\title{
FREE HAND VERSUS PARTIAL LIMITING DESIGN COMPUTER GUIDED SURGICAL STENT. (VITRO STUDY)
}

\author{
Mahmoud El Moutassim Bellah El Homossany*
}

\begin{abstract}
Aim of the study: Was to compare the accuracy of two different surgical techniques; free hand and partial limiting, regarding the accuracy of implant position.

Materials and methods: Four edentulous dummy mandible simulating human edentulous mandible were used (the model material duplicates type II III hardness). A surgical stent was fabricated. The surgical stent was secured on the first and second mandibles and all the implants were placed using the partial limit technique; where the initial drill was used first using the stent. The subsequent drills were used free hand. The same stent was placed on the other two mandibles where the position of the implants was delineated using a marker, then the implants were placed free hand. The angles between the long axis of preplanned and real implants were recorded from both the preoperative implant plan \& the post-operative implant representations on the resliced CBCT image \& were compared to each other in degrees. The linear deviation of the post-operative placement was recorded in $\mathrm{mm}$ in coronal \& apical positions from the pre-planned implants in both Mesio-distal \& Bucco-lingual aspects, also the vertical linear deviation (deviation in depth), was recorded apically, taking the pre-operative implant plan as the reference by the aid of the software matching function.
\end{abstract}

Results : The data was collected and statistically analyzed. The mean angular deviation for group A the free hand was $3.71 \pm 2.58$ degree while that for group B partial limiting was $1.72 \pm 1.67$ degree, this difference was statistically significant $\mathrm{P}<0.05$. The mean buccolingual deviation for group A was $0.79 \pm 0.66 \mathrm{~mm}$, while that for group B was $0.27 \pm 0.24 \mathrm{~mm}$, this difference was statistically significant $\mathrm{P}<0.05$. The mean Mesio distal deviation was $0.94 \pm 0.75 \mathrm{~mm}$ while that for group $\mathrm{B}$ was $0.37 \pm 0.35 \mathrm{~mm}$, this difference was statistically significant $\mathrm{P}<0.05$. For the mean apical deviation for group A it was $0.51 \pm 0.48 \mathrm{~mm}$ and that for group B was $0.32 \pm 0.32 \mathrm{~mm}$ and this was not statistically significant. $\mathrm{P}>0.05$. There was statistical significance difference between both groups concerning the angular deviation, Bucco lingual deviation and Mesio distal deviation, while apical deviation was not significantly different.

Conclusion: Partial limiting surgical stent is more precise than free hand surgical technique concerning the angular deviation, Bucco lingual deviation and Mesio distal deviation. There is no statistical significant difference concerning apical position.

* Lecturer Removable Prosthodontics Department, Faculty of Dentistry, Ain Shams University. 


\section{INTRODUCTION}

Osseointegrated implants are a practical alternative to traditional prosthodontics; however, designing an implant-supported prosthesis with function and esthetics is a challenge. ${ }^{1}$

Misaligned implants complicate the laboratory work of superstructures construction. Due to improper load distribution, an increase in stress on supporting structures may occur. This may compromise the bone level. A stent is an appliance used for radiographic evaluation of the available bone during treatment planning for correct implant placement. $^{2}$

With the use of computed tomography and computer added designing / computer added machining technology, it is possible to construct a surgical guide that allows the clinician to predetermine ideal locations virtually and surgically place them without tissue flap.

\section{Prosthodontically driven implant placement}

Earlier dentists placed implants where the greatest amount of bone was present, with less regard to placement of the final definitive restoration. Sometimes, the placement of the implant is not as accurate as intended. Even a minor variation in comparison to ideal placement causes difficulties in fabrication of final prostheses. ${ }^{2,3}$

Failures arise because of lack of consideration of the super structure during pre-surgical planning. Accurate placement is required to achieve best functional and esthetic result. An increase degree of precision in placement of the implant is very critical for the success of the appliance. This can be done by the aid of a surgical guide which provides accurate information regarding implant placement, and at the time of operation, it is adapted on to the existing dentition or on to the edentulous span. ${ }^{3,4}$

It was stated that no. of factors are involved in attaining a successful result for an implant supported prosthesis In these, the main factor is a placement of implant fixture in buccolingual, mesiodistal and apicocoronally dimension. The functions of final restoration are strongly affected by the placement of implant fixture. A small change in implant position can create a lot of problem for restoring prosthodontist. ${ }^{5}$

The 3-dimensional positioning of a dental implant is a key factor in attaining an adequate esthetic result. The position of the implant dictates the emergence profile of the tooth to be replaced; for this reason, implants should be positioned properly in all three spatial directions. Furthermore, achieving a long-lasting esthetic outcome requires using the final restoration as the guide for implant placement and considering the form and position of the planned prosthesis for final restoration. ${ }^{6}$

Over the past decade, advances in implant dentistry have helped create a greater appreciation for the esthetic demands of the clinician and the patient. $^{7}$

Because of these demands, implant dentistry has experienced a profound shift: from the function, with a surgically driven approach, to esthetics, with a prosthetically and biologically driven approach. In nature, what looks good usually works well. Applying this same premise to implant dentistry will allow a treatment outcome that balances esthetics with function. The ideal positioning of an implant in all three dimensions, regardless of the implant system used, has been well described in the dental literature. Published reports have also described zones of comfort and danger in the placement of an implant in the esthetic zone. ${ }^{8}$

Mesiodistally, the danger zones are located next to adjacent teeth. The facial danger zone is located anywhere facially to the imaginary line highlighted from the Point of the emergence of the adjacent teeth. The palatal danger zone begins $2 \mathrm{~mm}$ from the point of emergence and is associated with an increased risk of ridge lap restoration. ${ }^{8}$ 
Several guidelines have been suggested for optimizing esthetic results in implant placement. First, the position of the implant depends on the planned restoration that the implant will support. Second, the implant platform should be located 3 $\mathrm{mm}$ apical to the zeniths of the predetermined facialgingival margins of the planned restorations. Third, the center of the implant should be placed at least $3 \mathrm{~mm}$ palatal to the anticipated facial margins. The objective is to avoid poor facial bone thickness and gingival recession. Special consideration should be given to the thin gingival biotype; in such cases, it may be necessary to place the body and shoulder of the implant slightly more palatally to mask any show-through of titanium. ${ }^{8}$

Fourth, an inter implant spacing of $3 \mathrm{~mm}$ is required between adjacent implant platforms. A decrease in this spacing can cause resorption of the interproximal alveolar crest and a reduction in papillary height. Fifth, the implants should emerge through the palatal incisal edge of the ensuing crown positions. ${ }^{8}$

The risk of esthetic failure is higher when implants are placed "free hand," without surgical guides.

Reverse planning, starting from the final tooth position, allows determination of the exact location of the implant and assures an esthetic outcome. ${ }^{9,10}$

Careful planning and the use of a wax-up, a mock-up, and surgical guides will provide the surgeon with references for locating the implant properly in the three directions of the space: apico occlusal, mesiodistal, and labio palatal. In this manner, an esthetic result Can be achieved. However, fabricating a guide from a wax-up is associated with certain limitations. If the planned position does not match the available bone, the clinician has few options for making small changes. The recent development of virtual restorative planning is promising because it combines the ideal prosthetic position with the availability of bone.
Computer technologies, applied with knowledge, make esthetic complications unlikely and provide optimal function and appearance. ${ }^{11,12}$

The failures that result from improper implant placement are many and can lead to all the abovementioned pink-tissue failures. Nevertheless, these failures can be avoided by thorough treatment planning, careful site development, the use of surgical guides, and a proper understanding of restorative aspects when the implant is placed. ${ }^{13}$

\section{Partial limiting design}

A stent provided by the restorative dentist was used indicating ideal implant location. The stent, with imbedded metal bearings, is worn by the patient during CBCT. The image provides a more accurate image of the quantity and quality of the bone. The same template may be used as a surgical stent to help the surgeon in initial drill placement. Through careful planning, the predictable placement of implants can be achieved. With cooperative efforts of restorative dentists and surgeons, more than 400 implants have been successfully placed at University of California, Los Angeles, and School of Dentistry. ${ }^{14}$

A new implant placement surgical guide was described that gives implant location in $3 \mathrm{D}$ to the surgeon. Radiopaque markers are inserted on diagnostic dentures, and a lateral cephalometric radiograph is made that shows the osseous anatomy at syphilis and the anterior tooth location. The ideal implant location and trajectory data are transferred to a surgical stent that directs the angle and location of the implant at time of surgery. ${ }^{15}$

A novel guide template technique for implant placement was presented. This method may relieve some of the hazards common to conventional template techniques, where the drill is usually instantly guided by the template. The idea is to fixate the implant position and angulation by the help of Kirschner wires which are implanted in the alveolar 
ridge right through the mucosa before the rise of a mucoperiosteal flap. Thanks to this sequence, the template for guiding the wires may be placed precisely, even on grafted edentulous ridges. After the placement of the wires, the bone is exposed and the implant cavities prepared with a trephine drill guided over the wires alone or over wires combined with a special guidance cylinder fitting the trephine drill. The method may be combined with different planning and radiological techniques. Since the use of the template is detached from the actual drilling process, the danger of introducing debris of plastic or metal into the preparation site may be avoided. This technique might be helpful for difficult cases by improving communication between prosthodontist and surgeon and provide additional security to the surgeon with less time in dental implant insertion. ${ }^{16}$

Bilaminar dual-purpose stent was fabricated that facilitates ease in implant placement with improved verification of implant positioning. The outer lamina is designed for use in the computed tomography evaluation, using radiopaque markers. The verification of implant alignment and positioning, according to the determined prosthesis, is also performed with this template after modifying it for surgery. The inner lamina is designed to accept two removable surgical acrylic resin stents with different guide channels that avoid the risk of surgical malpractice. ${ }^{17}$

A technique that facilitates precise dental implant placement was described. A barium-coated stent with external guide wires used in conjunction with a computed tomography scan and interactive software may provide superior pre-surgical diagnostics, treatment planning, and prosthetically directed implant insertion. Measurements decided on the computed tomography scan can be assigned accurately to the diagnostic/surgical template by use of a precision milled cylinder placed into the template at the proper angulation and linear dimensions. The diagnostic/surgical template gives the surgeon the optimal position for implant placement, thus establishing greater clinical confidence when placing implants. ${ }^{18}$

Fabrication of a diagnostic template was described incorporating a silicone radiopaque marker as a guide for achieving the 3-dimensional evaluation of bone without artifacts using computed tomography. The diagnostic template may be easily transformed into a surgical template by removing the silicone marker. ${ }^{19}$

An acrylic resin implant placement guide was described which is simple to fabricate. This device guides the surgeon in the precise position and angulation planned for the implant yet allows for some flexibility in the event slight adjustments are necessary during surgery. ${ }^{20}$

A surgical template was fabricated that allowed the use of staged tooth extraction to help expected immediate implant insertion in a patient with a nonrestorable dentition. ${ }^{21}$

\section{- Fabrication of Stereolithographic Templates}

A computer-controlled, indirect milling machine was developed for reproduction of an anatomical structure $^{22}$. Further development resulted in a rapid prototyping of stereolithography. A rapid prototyping machine which uses the principle of stereolithography is employed to fabricate the stereolithographic models. The SLA consists of a vat which contains a liquid photo polymerized resin. A laser which is mounted on top of the vat moves in sequential cross-sectional increments of 50microns, which correspond to the slice intervals which are specified during the CT formatting fashion. The laser polymerizes the surface layer of the resin on contact. Once the first slice is performed, a mechanical table which is below the surface, moves down 50 microns, carrying with it the previously polymerized resin layer of the model.

The laser now polymerizes the next layer which is above the previously polymerized layer. In this manner, a complete stereolithographic model of the patient's jaw can be created. 
Approximately $80 \%$ of the total polymerization is completed in the vat; the remaining $20 \%$ can be achieved in a conventional ultraviolet light curing unit. $^{23,24}$

\section{MATERIALS AND METHODS}

Four edentulous dummy mandibles were used simulating human edentulous mandible are used (the model material duplicates type II III hardness). Four mandibles were radiographed using CBCT machine**. Standardization during imaging was achieved through adjusting the mandible positioning laser beams as follows. The seat height was adjusted to position the region of interest (ROI) vertically within the field of view (FOV). The upper laser beam indicated the top of the FOV and the lower laser beam indicated the bottom of the FOV.

The sagittal laser beam (vertical front beam) was positioned in the center of the FOV from sagittal direction so that it is in the center of the ROI. The lateral laser beam (vertical side beam) was positioned in the center of the FOV in the lateral direction so that it is in the center of the ROI. Exposure was performed at $15 \mathrm{~mA}, 85 \mathrm{KV}$ and at a field of view $7 \mathrm{~cm} \times 14.5 \mathrm{~cm} \times 14.5 \mathrm{~cm}$ for radiographing the mandible.

Image acquisition of the data was automatically by the computer then transferred via Ethernet connection to the work station for reconstruction by special software ${ }^{* * *}$ (Ondemand 3D, version 1.0.9, Cybermed, Korea). Image reconstruction was performed including axial, sagittal, coronal and 3D views .

On the axial cut the coronal orientation line was adjusted to be perpendicular on the long axis of the ridge in bucco-lingual direction, (called corrected coronal cut or cross-sectional cut) The sagittal orientation line was adjusted on the axial view to pass through the long axis of the mesio-distal dimension of the ridge (called corrected sagittal cut).

Linear measurements were recorded on the crosssectional view including bone height and buccolingual bone width. Virtual implant simulation with a suitable sized implant was selected $(3.7 \mathrm{~mm}$ diameter and $13 \mathrm{~mm}$ length), surrounded by at least $1 \mathrm{~mm}$ of bone bucco-lingually. Implant planning was performed on the software ${ }^{* * * *}$ using the preoperative CBCT, 8 Implants for each mandible were planned in the center of the alveolar ridge of the model, and for each quadrant.

\section{Surgical guide fabrication}

A surgical guide was fabricated according to the previous plan and position of the fixation pins was determined (three pins, one in the midline and one posterior for each side). The guiding sleeve $(5 \mathrm{~mm}$ diameter) is planned in the correct side. (figure 1) virtual surgical guide converted to an stl format on the same software for 3D printing using a 3D printer $^{* * * * *}$

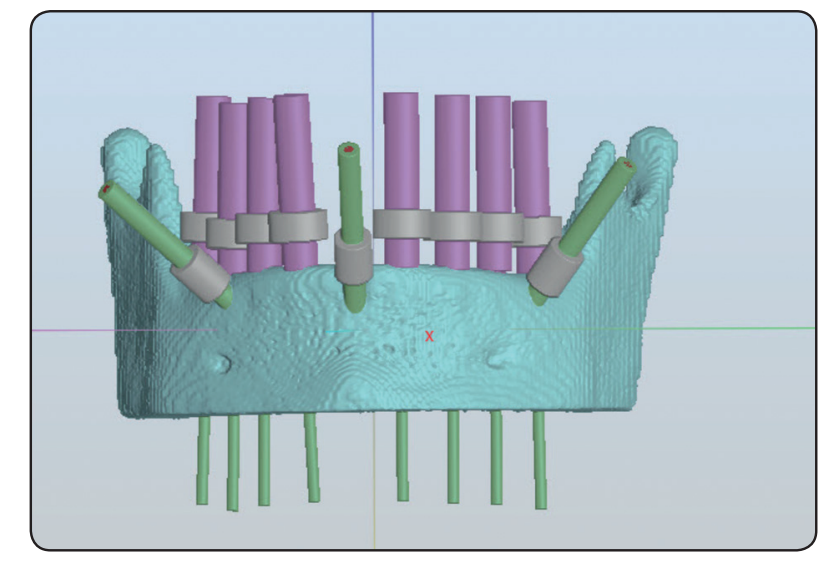

Fig. (1) Showing anterior view for preplanned implant position with planned guiding sleeves and planned fixation pins

\footnotetext{
* 1 IMP1003-L-HD, Nissin, japan

** Scanora 3D Soredex, Helsinki, Finland

*** Ondemand3D, version 1.0.9, Cybermed, Korea

*****3 diagnosys version 4.1, Italy

***** Envision tec Micro DGP, Germany
} 
The stereolithographic apparatus (SLA) consists of a vat which contains a liquid photo polymerized resin. A laser which is mounted on top of the vat moves in sequential cross sectional increments of 50 microns, which correspond to the slice intervals which are specified during the CT formatting procedure. The laser polymerizes the surface layer of the resin on contact. Once the first slice is completed, a mechanical table which is immediately below the surface, moves down 50 microns, carrying with it the previously polymerized resin layer of the model.

The laser now polymerizes the next layer which is above the previously polymerized layer. In this manner, a complete stereolithographic model of can be created.

Approximately $80 \%$ of the total polymerization is completed in the vat; the remaining $20 \%$ was completed in ultra-violet light curing unit.

The SL machine also reads the diameter and angulation of the simulated implants and it selectively polymerizes resin which is around them, forming a cylindrical guide which corresponds to each implant. A technician removed supporting resin triangles and connects surgical grade stainless steel tubes ( $5 \mathrm{~mm}$ internal diameter) into the cylindrical guide. In this manner, surgical template which seat directly on the dummy mandible and have metal sleeves which correspond to each fixture site had been generated.

The template itself had been fabricated from methacrylate resin (e-shell 600, Envisiontec, Germany). Which is a photo-polymerized resin and it has been FDA-approved to accurately guide the osteotomy drills.

\section{Implant placement}

The guided stent is secured in place with guide pins ( 3 pins one in the midline, one on the left

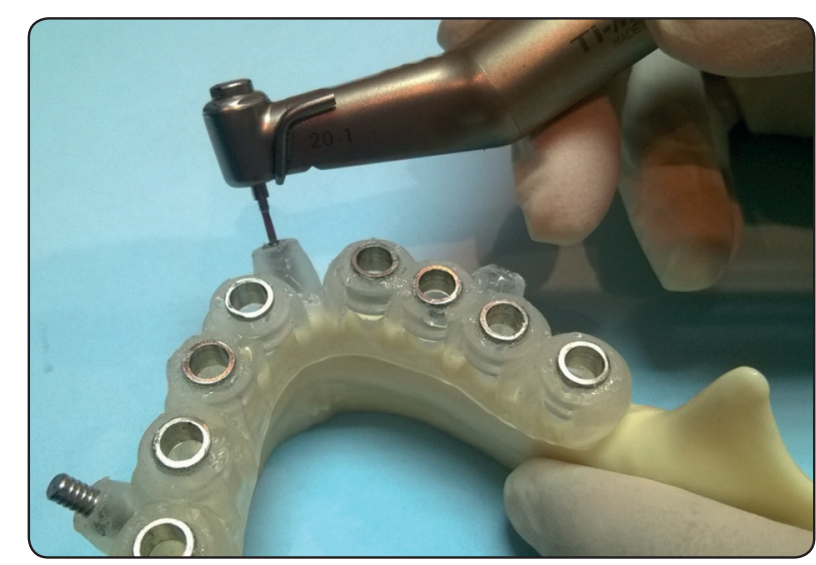

Fig. (2) Surgical guided stent in place secured with pins.

posteriorly and one in the right posteriorly) after drilling its holes (figure 2).

Eight implants are inserted for each jaw. Four implants for each quadrant. Drilling was started with the left side and for each quadrant from posterior to anterior. The non-limiting design (free hand) was achieved by drilling using the full sets without the computer guided surgical stent in place and their place were marked with black mark and the implant placed using this design were named group (A). The partially limiting design was achieved by using the initial drill when the guided stent in place and the remaining drills were used free hand (after removal of the surgical guide). The implants placed using this design were named group (B). The implants used have the same length $(13 \mathrm{~mm})$ and the same diameter (3.7) * and the drilling was done by using the surgical guide drills which has shank and guide stop ${ }^{* *}$, beginning with initial drill then $(2.3 \times 13),(2.8 \times 13),(3.2 \times 13)$ and finally $(3.7 \times 13)$.

\section{Postoperative cbct and deviation measurement}

Post-operative CBCT was performed \& the CBCT image was resliced, taking the preoperative CBCT image (the taking the whole mandible bone) as a reference to allow standardization in the same

\footnotetext{
* Spectra, implant direct, USA

** Ekram smart kit, M. Ekram imaging, Egypt
} 
position as the preoperative CBCT by the aid of the software matching function

The angles between the long axis of preplanned and real implants were recorded from both the preoperative implant plan \& the post-operative implant representations on the resliced CBCT image $\&$ were compared to each other in degrees.

The linear deviation of the post-operative placement was recorded in $\mathrm{mm}$ in coronal \& apical positions from the pre-planned implants in both mesio-distal \& bucco-lingual aspects, also the vertical linear deviation (deviation in depth), was recorded apically, taking the pre-operative implant plan as the reference by the aid of the software matching function. Figure 3

Numerical data were explored for normality by checking the data distribution, calculating the mean and median values, evaluating histograms and normality curves and using Kolmogorov-Smirnov and Shapiro-Wilk tests.

Data were assumed normally distributed and

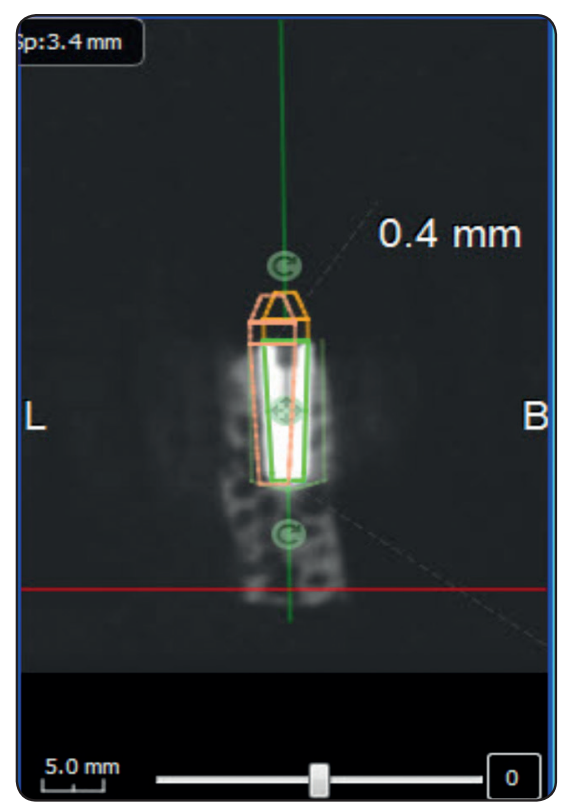

were presented as mean and standard deviation. Unpaired $\mathrm{t}$ test was used for comparison between groups The significance level was set at $\mathrm{P} \leq 0.05$. Statistical analysis was performed with IBM $^{\circledR}$ SPSS $^{\circledR}$ Statistics Version 20 for Windows.

\section{RESULTS}

The mean angular deviation for group A the free hand was $3.71 \pm 2.58$ degree while that for group B partial limiting was $1.72 \pm 1.67$ degree, this difference was statistically significant $\mathrm{P}<0.05$. The mean buccolingual deviation for group A was $0.79 \pm 0.66 \mathrm{~mm}$, while that for group B was $0.27 \pm$ $0.24 \mathrm{~mm}$, this difference was statistically significant $\mathrm{P}<0.05$. The mean Mesio distal deviation was $0.94 \pm 0.75 \mathrm{~mm}$ while that for group B was $0.37 \pm$ $0.35 \mathrm{~mm}$, this difference was statistically significant $\mathrm{P}<0.05$. For the mean apical deviation for group $\mathrm{A}$ it was $0.51 \pm 0.48 \mathrm{~mm}$ and that for group $\mathrm{B}$ was 0.32 $\pm 0.32 \mathrm{~mm}$ and this was not statistically significant. $\mathrm{P}>0.05$
Fig. (3) Show the linear deviation of postoperative placement was recorded in $\mathrm{mm}$ in coronal and apical positions from the preplanned implants in both mesiodistal and buccolingual aspects. Also the vertical linear deviation (deviation in depth was statistically analyzed)(the green line mark the actual implant and the orange one mark the preplanned virtual implant). 
TABLE (1) Showing deviation between the two groups

\begin{tabular}{|c|c|c|c|c|c|}
\hline Group & $\begin{array}{c}\text { Number of } \\
\text { implants }\end{array}$ & $\begin{array}{c}\text { Angular deviation } \\
\text { (degrees } \mathrm{M} \pm \mathrm{SD})\end{array}$ & $\begin{array}{c}\text { Bucco lingual deviation } \\
(\mathrm{mm}, \mathrm{M} \pm \mathrm{SD})\end{array}$ & $\begin{array}{c}\text { Mesio distal } \\
(\mathrm{mm}, \mathrm{M} \pm \mathrm{SD})\end{array}$ & $\begin{array}{c}\text { Apical deviation } \\
(\mathrm{mm}, \mathrm{M} \pm \mathrm{SD})\end{array}$ \\
\hline $\begin{array}{c}\text { Free hand } \\
\text { (Group A) }\end{array}$ & 16 & $3.71 \pm 2.58$ & $0.79 \pm 0.66$ & $0.94 \pm 0.75$ & $0.51 \pm 0.48$ \\
\hline $\begin{array}{c}\text { Partial limiting } \\
\text { (Group B) }\end{array}$ & 16 & $1.72 \pm 1.67$ & $0.27 \pm 0.24$ & $0.37 \pm 0.35$ & $0.32 \pm 0.32$ \\
\hline P value & & 0.0147 & 0.0059 & 0.0099 & 0.1977 \\
\hline
\end{tabular}

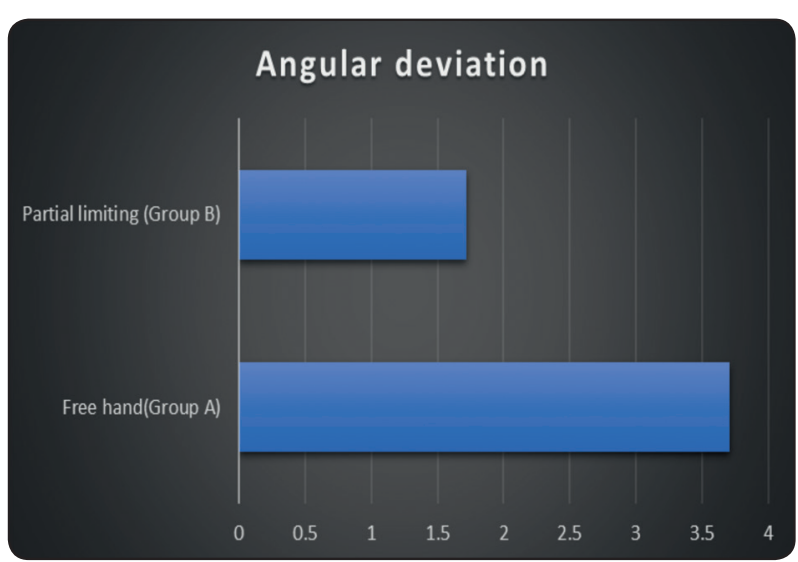

Fig. (4) Bar graph showing angular deviation in degrees

\section{DISCUSSION}

\section{Discussion of material and method}

Four dummy mandible to stimulate human mandible were used .This type of dummy mandible was used because its material duplicates type II III hardness of bone( plastic model manufactured by layering) which is more common and can appear obviously in cbct alone and with implant in place.

CBCT can be used to measure the distance between 2 points in alveolar bone ${ }^{25}$

In our study, the models were radiographed using CBCT machine (Scanora 3D Soredex, Helsin ki, Finland) which show good accuracy since the image data produced by the cone beam CT machine is isotropic, thus the $3 \mathrm{D}$ volume elements are cubical. ${ }^{26}$

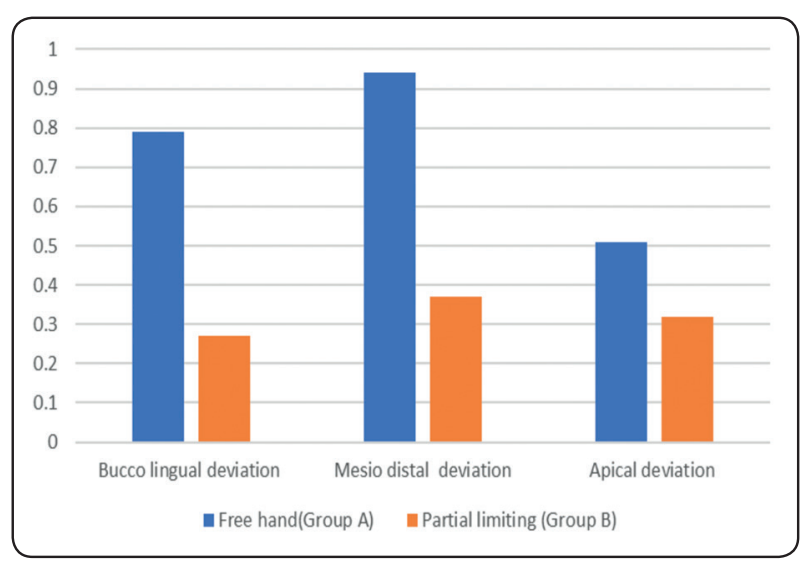

Fig. (5) Showing bar graph of the deviations between the groups in $\mathrm{mm}$.

Stereolithographic surgical guide stent was used in this study due to its high accuracy compared to conventional stents. A study has shown that the average distance between the planned implant and the actual osteotomy was $1.5 \mathrm{~mm}$ at the entrance and $2.1 \mathrm{~mm}$ at the apex when the conventional surgical guide was used, The same measurements were significantly reduced to $0.9 \mathrm{~mm}$ and $1.0 \mathrm{~mm}$ when the stereolithographic surgical guide was used. ${ }^{27}$

Conventionally fabricated surgical templates have certain limitations in achieving optimal results. When fabricated on diagnostic study casts, the soft tissue is a rigid, nonfunctional representation and does not provide information about the varying thickness of the mucosa, topography of underlying bone, or vital anatomical structures that lie within. In addition, the limitations of 
conventional dental radiography about dimensional accuracy and inability to visualize anatomical structures in parasagittal sections further hinder accurate evaluation. For completely edentulous cases, conventional guide may interfere with flap reflection but computer guided surgical stent can be made bone supported from begging.

In this study, simulated flap technique was done. The surgical guide was fabricated on bone not mucosa to exclude viscoelasticity of mucosa and bone supported guide will seat more precise than mucosa supported one.

The template itself had been fabricated from methacrylate resin (e-shell 600, Envisiontec, Germany). Which is a photo-polymerized resin and it has been FDA-approved to accurately guide the osteotomy drills. ${ }^{27,28}$

Fixing pins (one in the midline and one in each side posteriorly right and left) were used to stabilize the guide during drilling. The sleeves used were compatible with the drills.

Evaluation of accuracy is done by superimposition of pre and postoperative cbct by the aid of matching function of the software(by taking the preoperative whole mandible bone as a reference). ${ }^{29}$

\section{Discussion of results}

Researches had found great accuracy of complete limiting design of computer guided surgical stent compared to non-limiting design. The difference between planned and actually placed implant in the maxilla were at the cervical, middle, and apical implant portions of $2.17( \pm 0.87), 2.32( \pm 1.52)$, and $2.86( \pm 2.17) \mathrm{mm}$ respectively . ${ }^{30}$

Also, in a review of accuracy in computer-guided surgery a maximum planning vs. actual position difference between 1.2 and $2 \mathrm{~mm}$ were found .2 Van Assche et al. use cone beam tomography and stereolithographic templates to drill and insert 12 dental implants into four cadaver jaws, a shoulder linear deviation of $+/-1.10 .7 \mathrm{~mm}^{31}$, an apex linear deviation of $2+/-0.7 \mathrm{~mm}$, (range $0.7-2.4 \mathrm{~mm}$ ) and an angular deviation of $2+/-0.8^{\circ}$ (range $0.7-41$ ) were found.

In an in vitro study evaluating the accuracy of three different computer-aided surgery systems, the SimPlant/SurgiGuide system was found to have mean depth deviations of0.6 -/+ $0.4 \mathrm{~mm}$, mean radial deviations of $1.5-/+0.8 \mathrm{~mm}$ and mean angular deviations of $7.9+/-5^{\circ} .32$

But in our study, we compare partial limiting design with non-limiting both at buccolingual (important to avoid buccal or lingual bone perforation), mesiodistal (important to avoid injury of neighboring teeth or implant) linear and angular deviation, and apical linear deviation (important to avoid injury of vital structure as maxillary sinus or inferior alveolar nerve).

While the risk of deviation and errors remains, $\mathrm{CAD} / \mathrm{CAM}$ surgical guides have led to optimal clinical results because of the efficacy and ease-ofuse of this technology. Compared with conventional implantation techniques, the use of CAD/CAM surgical guides result in improved precision in terms of position, angulation, and depth of implant placement. One limitation is the radiation exposure during pre- and postoperative CT scanning, which is required to evaluate the precision of planned and placed implants. Clinicians should carefully assess any risks to patients before surgery. ${ }^{33}$

The results of this study go with Nickenig et al, they found an average positional precision of $\leq 1 \mathrm{~mm}$ and within 5 degrees of deviation in inclination can be achieved. Their study documented that the axis and implant position is significantly more precise when using a three-dimensional surgical guide compared to freehand placement. ${ }^{34}$ 


\section{REFRENCES}

1. Verde, M.A. and S.M. Morgano, A dual-purpose stent for the implant-supported prosthesis. J Prosthet Dent, 1993. 69(3): p. 276-80.

2. Widmann, G. and R.J. Bale, Accuracy in computer-aided implant surgery--a review. Int J Oral Maxillofac Implants, 2006. 21(2): p. 305-13.

3. Takeshita, F. and T. Suetsugu, Accurate presurgical determination for implant placement by using computerized tomography scan. J Prosthet Dent, 1996. 76(6): p. 590-1.

4. Chiu, W.K., W.K. Luk, and L.K. Cheung, Three-dimensional accuracy of implant placement in a computer-assisted navigation system. Int J Oral Maxillofac Implants, 2006. 21(3): p. 465-70.

5. Sadig, W., A comparative in vitro study on the retention and stability of implant-supported overdentures. Quintessence Int, 2009. 40(4): p. 313-9.

6. Martin, W.C., A. Pollini, and D. Morton, The influence of restorative procedures on esthetic outcomes in implant dentistry: a systematic review. Int J Oral Maxillofac Implants, 2014. 29 Suppl: p. 142-54.

7. Al-Sabbagh, M., Implants in the esthetic zone. Dent Clin North Am, 2006. 50(3): p. 391-407, vi.

8. Buser, D., W. Martin, and U.C. Belser, Optimizing esthetics for implant restorations in the anterior maxilla: anatomic and surgical considerations. International Journal of Oral \& Maxillofacial Implants, 2004. 19(7).

9. $\operatorname{lmog}$, D.M., E. Torrado, and S.W. Meitner, Fabrication of imaging and surgical guides for dental implants. J Prosthet Dent, 2001. 85(5): p. 504-8.

10. Noharet, R., A. Pettersson, and D. Bourgeois, Accuracy of implant placement in the posterior maxilla as related to 2 types of surgical guides: a pilot study in the human cadaver. J Prosthet Dent, 2014. 112(3): p. 526-32.

11. De Almeida, E.O., et al., Computer-guided surgery in implantology: review of basic concepts. J Craniofac Surg, 2010. 21(6): p. 1917-21.

12. Katsoulis, J., P. Pazera, and R. Mericske-Stern, Prosthetically driven, computer-guided implant planning for the edentulous maxilla: a model study. Clin Implant Dent Relat Res, 2009. 11(3): p. 238-45.

13. Chee, W. and S. Jivraj, Failures in implant dentistry. Br Dent J, 2007. 202(3): p. 123-9.
14. Engelman, M.J., J.A. Sorensen, and P. Moy, Optimum placement of osseointegrated implants. J Prosthet Dent, 1988. 59(4): p. 467-73.

15. Adrian, E.D., J.R. Ivanhoe, and W.A. Krantz, Trajectory surgical guide stent for implant placement. J Prosthet Dent, 1992. 67(5): p. 687-91.

16. Minoretti, R., B.R. Merz, and A. Triaca, Predetermined implant positioning by means of a novel guide template technique. Clin Oral Implants Res, 2000. 11(3): p. 266-72.

17. Cehreli, M.C., A.C. Calis, and S. Sahin, A dual-purpose guide for optimum placement of dental implants. J Prosthet Dent, 2002. 88(6): p. 640-3.

18. Kopp, K.C., A.H. Koslow, and O.S. Abdo, Predictable implant placement with a diagnostic/surgical template and advanced radiographic imaging. J Prosthet Dent, 2003. 89(6): p. 611-5.

19. Tsuchida, F., et al., A technique for making a diagnostic and surgical template. J Prosthet Dent, 2004. 91(4): p. 395-7.

20. Windhorn, R.J., Fabrication and use of a simple implant placement guide. J Prosthet Dent, 2004. 92(2): p. 196-9.

21. Al-Harbi, S.A. and R.G. Verrett, Fabrication of a stable surgical template using staged tooth extraction for immediate implant placement. J Prosthet Dent, 2005. 94(4): p. 394-7.

22. D'souza, K.M. and M.A. Aras, Applications of computeraided design/computer-assisted manufacturing technology in dental implant planning. Journal of Dental Implants, 2012.2(1): p. 37.

23. Sarment, D.P., P. Sukovic, and N. Clinthorne, Accuracy of implant placement with a stereolithographic surgical guide. International Journal of Oral \& Maxillofacial Implants, 2003. 18(4).

24. Ozan, O., et al., Clinical accuracy of 3 different types of computed tomography-derived stereolithographic surgical guides in implant placement. J Oral Maxillofac Surg, 2009. 67(2): p. 394-401.

25. Brown, A.A., et al., Linear accuracy of cone beam CT derived 3D images. Angle Orthod, 2009. 79(1): p. 150-7.

26. Hassan, B., et al., Comparison of five cone beam computed tomography systems for the detection of vertical root fractures. J Endod, 2010. 36(1): p. 126-9.

27. Sarment, D.P., P. Sukovic, and N. Clinthorne, Accuracy of implant placement with a stereolithographic surgical 
guide. International Journal of Oral \& Maxillofacial Implants, 2003. 18(4).

28. Lal, K., et al., Use of stereolithographic templates for surgical and prosthodontic implant planning and placement. Part I. The concept. Journal of Prosthodontics, 2006. 15(1): p. 51-58.

29. Horwitz, J., O. Zuabi, and E.E. Machtei, Accuracy of a computerized tomography-guided template-assisted implant placement system: an in vitro study. Clinical oral implants research, 2009. 20(10): p. 1156-1162.

30. Vieira, D.M., et al., Clinical accuracy of flapless computerguided surgery for implant placement in edentulous arches. International Journal of Oral \& Maxillofacial Implants, 2013. 28(5).

31. Horwitz, J., O. Zuabi, and E.E. Machtei, Accuracy of a computerized tomography-guided template-assisted im- plant placement system: an in vitro study. Clin Oral Implants Res, 2009. 20(10): p. 1156-62.

32. Ruppin, J., et al., Evaluation of the accuracy of three different computer-aided surgery systems in dental implantology: optical tracking vs. stereolithographic splint systems. Clin Oral Implants Res, 2008. 19(7): p. 709-16.

33. Cassetta M, Giansanti M, Di Mambro A, Calasso S, Barbato E. Accuracy of Two Stereolithographic Surgical Templates: A Retrospective Study. Clin Implant Dent Res 2013; 15: 448-459.

34. Nickenig, H.J., Eitner, S., Rothamel, D., Wichmann, M., \& Zöller, J.E. (2012) Possibilities and limitations of implant placement by virtual planning data and surgical guide templates. International Journal of Computerized Dentistry, 15, 9-21. 